G C, Deepak, Ladas, Alexandros and Politis, Christos (2019) Robust Device-to-Device 5G Cellular Communication in the Post-Disaster Scenario. In In: 2019 16th IEEE Annual Consumer Communications \& Networking Conference (CCNC). Piscataway, U.S. : Institute of Electrical and Electronics Engineers, Inc. ISSN (online) 2331-9860 ISBN 9781538655535. 


\title{
Robust Device-to-Device 5G Cellular Communication in the Post-Disaster Scenario
}

\author{
Deepak G. C., Alexandros Ladas, Christos Politis \\ School of Computer Science and Mathematics \\ Kingston University, London, KT1 2EE, United Kingdom \\ Email:\{d.gc, a.ladas, c.politis\}@kingston.ac.uk
}

\begin{abstract}
The wireless communication is an integral part of the society, however, wireless network infrastructure may not fully function during post-disaster wireless network scenario in which we need wireless services even more than normal circumstances. In such post-disaster scenarios, the base stations (BS) could possibly be dysfunctional or overloaded by an excessive number of user calls or data, whereas user terminals are likely to be partially connected to or fully isolated from the BS radio signals. In this paper, we study the network behavior during the post-disaster when BSs undergo the thinning process due to the damage to the network infrastructure. During network recovery phase, we also study the network performance when new temporary BSs are distributed which will bring the network into BS superposition phase. Furthermore, Device-to-Device (D2D) assisted cellular communication helps to increase the link level network connectivity which is highly appreciated during postdisaster network scenario because the network coverage is more desirable than the network throughout in such cases. We also present the analytical study of D2D and cellular communication and show that it will effectively increase the network coverage which may ultimately save many lives during the golden hours of post-disaster phase.
\end{abstract}

Index Terms-D2D communication, Poisson point process, public safety communication, network coverage, base stations thinning

\section{INTRODUCTION}

The broadband wireless networks, for instance, long-term evolution (LTE), and future 5G communication system have recently gained tremendous attention due to their capacity, reliability and spectrum efficiency. Therefore, we need to investigate how they may fulfill to the mission critical requirements of public safety communication, specifically, during and after the disasters occur [1]. As a result, the post-disaster communication network requires a robust, reliable, energy efficient and spectral efficient wireless network infrastructure. It is therefore obvious that we require advanced technologies that can efficiently operate under mission critical situations and retain coverage even when the infrastructure is partially damaged or completely unavailable during the golden hour of post-disaster scenario [2].

In LTE, the provision of enhanced multimedia priority service (eMPS) is available for public protection and disaster relief (PPDR). According to 3GPP TR 23.854 [3], eMPS functionality in LTE allows authorized users to obtain and maintain the radio resource and network components. Such facilities are provided during eMPS session, i.e., the duration in which such functionality is enabled in the network, where priority treatment is applied in resource allocation. Such priority sessions are basically anticipated on an end-to-end basis. However, in the post-disaster network scenario, the cellular services may be unavailable or partially available. In such cases, there must be fairness in resource allocation and satisfactory level of network connectivity such that every user can connect to the scarce radio resources.

One of the several promising technologies that are identified for $5 \mathrm{G}$ is the device-to-device (D2D) communication, where users are allowed to directly communicate without the base stations (BSs) involvement. The spectrum resources are shared between D2D and cellular communications through either overlay, i.e., orthogonal access to time/frequency resource, or underlay method, i.e., opportunistic access of time/frequency resources. It ultimately helps to increase the spectral and energy efficiencies due to lower transmit power [4]. Such features make D2D communication a very attractive solution for post-disaster network scenarios, where there is limited power supply and efficiency of BSs is critically compromised [5], [6].

In cases of normal situations, network capacity is expected to be maximum within the wireless resource constraints. However, network coverage is critically important than capacity during the post-disaster for users' communication to achieve the effective coordination of the search-and-rescue operations [7]. Furthermore, there must be absolute fairness on resource allocation among the end users because every user requires wireless network connection to the BSs during the postdisaster. Therefore, the D2D communication features should be investigated further to increase the coverage area such that a large number of users are covered within the communication range. Furthermore, the prototype of multihop D2D communications, by implementing relay-by-smartphone concept, has been investigated in [8], where the D2D communication is integrated with the routing techniques to realize a multihop communication to improve the network coverage.

Although D2D communication improves the energy and spectral efficiencies, it also negatively impacts the cellular network performance due to the severe co-channel interference. This ultimately degrades the QoS of both cellular and D2D services in cases when network is already fragile during post-disaster scenario. There are several transmit power control 


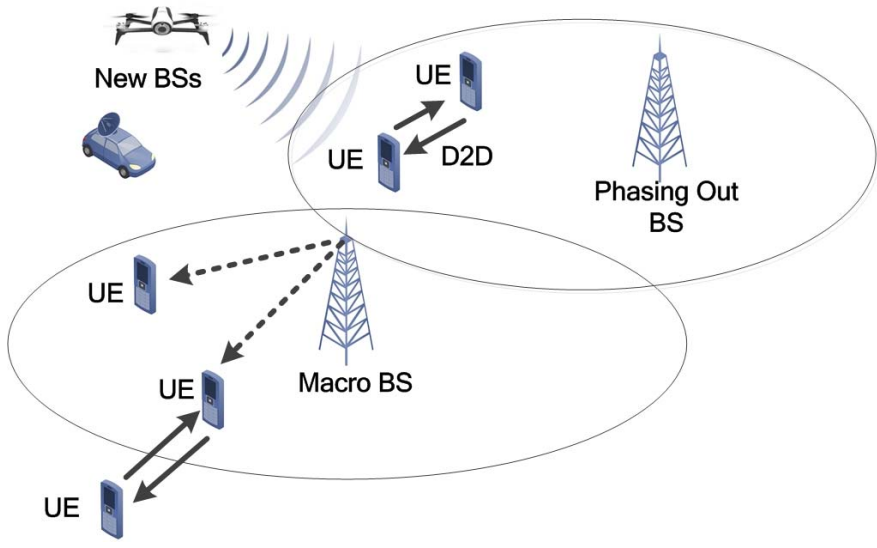

Fig. 1. The system model of the typical post-disaster communication scenario.

algorithms proposed in the literature, see, e.g., [9], [10], [11], to avoid interference from cellular to D2D network as well as D2D to cellular network. Such techniques are inevitable for efficient post-disaster communication framework, however, the complexity of algorithm should be significantly minimum to save energy for information processing in both BSs and user devices during the post-disaster.

There are several approaches to analyze the cellular and D2D networks. Stochastic geometry is relatively the most effective way to study the modern heterogeneous networks. Furthermore, there are very little studies on the behavior of network during post-disaster communication. The analytical study presented in this paper contributes to understand the post-disaster network behavior. Furthermore, it will also help to mitigate the co-channel interference, user association and radio resource allocation when the disaster hits the wireless network infrastructure.

\section{SySTEM MOdEL}

The network scenario consists of cellular BSs which are distributed according to the homogeneous Poisson point process (PPP), $\Phi_{b} \in \mathcal{R}^{2}$, of uniform intensity $\lambda_{b}$, where $\lambda_{b}$ is the average number of BSs per unit area and $\frac{1}{\lambda_{b}}$ is the area of the cell. When the disaster occurs, as shown in Fig. 1, the cellular users can terminate the cellular services in case of deteriorating quality of service $(\mathrm{QoS})$ on voice and data applications. The users thereafter can initiate the proximity services (ProSe) [3] to form a robust D2D communication among users. The users are assumed to be randomly distributed according to the stationary PPP, $\Phi_{u} \in \mathcal{R}^{2}$, with the intensity of $\lambda_{u}$ as following.

$$
\Phi_{u}=\left\{\delta_{i}, L_{i}, P_{i}\right\} .
$$

Here, $\left\{\delta_{i}\right\}_{i \in[c, d]}$, where $c$ and $d$ indicate cellular and D2D communication modes, respectively, is the indicator to show which mode of operation a user prefers when cellular network is partially available when the disaster occurs. It primarily depends on the received and threshold values of signal to noise and interference ratio (SNIR). Also, $\left\{L_{i}\right\}_{i \in[c, d]}$ is the quality of radio link and $\left\{P_{i}\right\}_{i \in[c, d]}$ is the transmit power of user equipment which is a random variable that primarily depends on $\left\{L_{i}\right\}$ and path loss exponent, $\alpha$. The users have to initiate the synchronization in time and frequency among users and BSs within the D2D hops to share resource blocks for D2D links. However, the users in D2D mode can also communicate independently without the BS assistance during post-disaster scenarios.

The transmit power can be estimated using the channel inversion technique in time division duplex (TDD) mode. The cellular network is based on the orthogonal frequency division multiple access (OFDMA) technology and BSs transmit with fixed power, $P_{t x}$, to the desired user on any optimal timefrequency resources.

In the considered network scenario, D2D communication among users is incorporated in cellular networks. The users can enable the mode selection between D2D and cellular during the post-disaster communication depending the user requirements and available resources. The aim of this paper is to develop an analytical model to study the behavior of D2D-enabled cellular network specifically for the postdisaster communication. Considering the complexity of the D2D communication within the cellular network, the users select D2D mode only when channel quality indicator (CQI) of cellular link is below the SNIR threshold $(\Theta)$, thereby creating a multihop D2D link to the next available BSs. Therefore, we consider that each D2D mode user is randomly and independently placed around another D2D mode user. From the definition of homogeneous PPP, the circular void probability is $\mathbb{P}(D \geq r)=\exp \left(-\lambda_{u} \pi r^{2}\right)$, where $D$ is the distance from the nearest point of the process from the random point. Here, the probability distribution function (PDF) of distance $D$ is obtained as follows.

$$
f_{D}(r)=2 \pi \lambda_{u} r \mathrm{e}^{-\lambda_{u} \pi r^{2}}, r \geq 0 .
$$

For instance, when the mode selection is ideally based on SNIR, cellular mode is used if SNIR $\geq \Theta$, where $\Theta$ is the threshold SNIR. Otherwise, D2D communication mode is selected when multi-hop or single-hop pair formation is successfully established.

\section{A. Spectrum Access Method}

The selection of spectrum sharing mode in D2D-enabled cellular network is very critical in post-disaster network scenario. The overlay mode, i.e., the $B$ subchannels are divided into $\eta B$, where $\eta \in[0,1]$, for $\mathrm{D} 2 \mathrm{D}$ communication and rest $(1-\eta) B$ for cellular communication. To maximize the spectral efficiency, $\eta$ can be varied in real-time and OFDMA resource blocks are managed by centralized system. However, it requires an optimal spectrum sensing techniques to improve the spectrum allocation efficiency [12]. Though such a mechanism has a lower sub-band interference, it may not be an ideal spectrum allocation technique in some situations, e.g., postdisaster scenario, where central system may be dysfunctional or partly functioning. 
Alternatively, underlay in-band D2D communication would be suitable for post-disaster communication where D2D users randomly and independently access part of OFDMA resource blocks, i.e., $B$, by calculating the optimal transmit power such that the interference to the other cellular or D2D users remains lower than the threshold, $\Theta$, which is a system defined parameter.

\section{Post-Disaster Network Behavior}

We assume that $\mathbf{M}=\left(M_{t}: t \geq 0\right)$ is the counting process which denotes a set of phasing out BSs in the interval $(0, t]$ when a disaster hits at any arbitrary time $t=0$. Similarly, counting process $\mathbf{N}=\left(N_{t}: t \geq 0\right)$ represents the functional BSs during interval $(0, t]$.

Lemma 1. The random phasing out of BSs, which are distributed according to homogeneous PPP, will result in a homogeneous PPP.

Remark 1. If $\mathbf{M}=\left(M_{t}: t \geq 0\right)$ follows PPP with parameter $p \lambda$, where $p \in[0,1]$ is the thinning factor which indicates the probability of any random $B S$ to be phased out during post-disaster, then $\mathbf{N}=\left(N_{t}: t \geq 0\right)$ follows PPP with the parameter $(1-p) \lambda$.

Theorem 1. The counting processes $\mathbf{M}=\left(M_{t}: t \geq 0\right)$ and $\mathbf{N}=\left(N_{t}: t \geq 0\right)$ are independent PPP.

Proof. Let us consider $j \in \mathcal{M}, k \in \mathcal{N}$. It is straightforward to obtain the following, which completes the proof.

$$
\mathbb{P}\left(M_{t}=j, N_{t}=k\right)=\mathrm{e}^{-p r t} \frac{[p r t]^{j}}{j !} \cdot \mathrm{e}^{-(1-p) r t} \frac{[(1-p) r t]^{k}}{k !},
$$

Therefore, when the disaster occurs and BSs start phasing out, the first task is to ensure the public safety by providing the additional infrastructure, for instance, by deploying the flying BSs [13] or initiating D2D communication mode to provide better connectivity irrespective of the exact pattern of phasing out BSs.

Lemma 2. During the post-disaster network, let us consider that $\mathbf{X}=\left(X_{t}: t \geq 0\right)$ is the current set of working $B S s$ and $\mathbf{Y}=\left(Y_{t}: t \geq 0\right)$ is the union set of additional $B S$ s deployed and D2D pairs formation, are two independent counting processes. Here, $\mathbf{X}$ and $\mathbf{Y}$ follow the homogeneous PPP with rates $\lambda_{1}$ and $\lambda_{2}$ to counteract the BSs thinning effect. The combination of $\mathbf{X}$ and $\mathbf{Y}$ during post-disaster recovery phase will also result in homogeneous PPP with the rate $\lambda_{1}+\lambda_{2}$, following the superposition property of PPP.

According to Lemma 2, when the new BSs, e.g., aerial BSs or ground-based vehicular BSs with sufficient backup power, are deployed to the already existing fragile postdisaster network, the new network can be analyzed according to homogeneous PPP.

In a typical post-disaster scenario, network thinning occurs due to the phasing out BSs, and at the same time, network superposition occurs due to the added network infrastructures to improve the network connectivity and throughput. However, it must be noted that both are independent and random events in real network because distribution of new BSs does not consider the actual thinning process in a typical post-disaster scenario. In the next section, we further investigate how the network distribution and its behavior change within golden hour of post-disaster.

Theorem 2. When BSs are phasing out according to homogeneous PPP with the parameter $p \lambda, p \in[0,1]$ and superposition occurs by $i=\{1, \ldots, K\}$ independent network infrastructure with the parameter $\lambda_{i}$, the new network will also follow the homogeneous PPP with the new parameter $\left(p \lambda+\sum_{i=1}^{K} \lambda_{i}\right)$.

Proof. see Appendix A.

\section{Measurement of Success Probability}

The received SNIR is an important parameter which represents the strength of the desired signal, $\mathbf{S}$, in comparison to the sum of interference, $\mathbf{I}$, and additive white Gaussian noise (AWGN), $W$, as follows.

$$
\mathbf{S N I R}=\frac{\mathbf{S}}{\mathbf{I}+W} .
$$

The link level successful communication primarily depends on the received SNIR, which determines the spectral efficiency, i.e., $\log _{2}(1+$ SNIR $)$, measured in $\mathrm{bps} / \mathrm{Hz}$. Therefore, for certain applications, the probability of successful transmission, $\mathbb{P}_{s}$, in both cellular and D2D mode is obtained as follows.

$$
\mathbb{P}_{s}=\mathbb{P}(\mathbf{S N I R} \geq \Theta) .
$$

The SNIR threshold, $\Theta$, is a system defined parameter which depends on the traffic pattern and mode of operation, i.e., cellular or D2D communication, when a disaster occurs.

As shown in Theorem 2, The post-disaster communication scenario can be studied following the properties of homogeneous PPP. In such cases, when BSs undergo phasing out mode immediately after the disaster, i.e., $\Phi_{b}(\lambda) \rightarrow \Phi_{b}(p \lambda \mid p \in$ $[0,1])$, or the superposition mode during the network recover process, i.e., $\Phi_{b}(\lambda) \rightarrow \Phi_{b}\left(\sum_{i} \lambda_{i}\right)$, the network performance can be obtained by considering the PPP model. In this paper, we adopt the path loss and Rayleigh fading propagation model in the wireless channels. We denote the direct communication to the cellular network event by $\mathbf{C}$ or through a D2D peer by D. According to PPP network analysis in [14], the probability of successful transmission during post-disaster when BS and users in cellular mode can be obtained as follows.

$$
\begin{aligned}
\mathbb{P}_{s}\left(C_{t}: t \geq 0\right) & =\exp \left(-\frac{r_{o}^{2 b} \Theta W}{P_{t x}}\right) \times \\
& \exp \left[-\frac{\pi \lambda_{I} C_{1 / b}^{-1} \Gamma\left(1+\frac{1}{b}\right)}{\cos \left(\frac{\pi}{2 b}\right)}\left(\frac{P_{I}}{P_{T}} r_{o}^{2 b} \Theta\right)^{\frac{1}{b}}\right] .
\end{aligned}
$$

Here, $C_{\alpha}$ is defined as

$$
C_{1 / b}^{-1} \triangleq \begin{cases}\frac{1-\alpha}{\Gamma(2-\alpha) \cos (\pi \alpha / 2)}, & \text { if } \alpha \neq 1 \\ \frac{2}{\pi}, & \text { if } \alpha=1\end{cases}
$$


where, $\Gamma(x)=\int_{0}^{\infty} y^{x-1} \mathrm{e}^{-y} d y$ denotes the Gamma function, $b$ is related to the channel propagation exponent as $b=1 / \alpha$, $P_{I}$ is the aggregated interference power and $\lambda_{I}$ is the density of interferer transmitters.

Similarly, the D2D mode is initiated when the SNIR from the cellular BS is negligible in comparison to the D2D peer. In such cases, the multihop D2D communication success probability [7] is obtained as follows.

$$
\begin{array}{r}
\mathbb{P}_{s}\left(D_{t}: t \geq 0\right)=\mathbb{P}\left(P_{T} \leftarrow \bar{P}_{T}, \alpha, P_{I} \leftarrow \bar{P}_{I}, \lambda_{I} \leftarrow \bar{\lambda}_{I},\right. \\
\left.\Theta \leftarrow \bar{\Theta}, r_{o}\right),
\end{array}
$$

The new communication parameters for D2D case, i.e., $A \leftarrow \bar{A}$ as indicated in (8), are selected based on the density of D2D users, SNIR threshold, fading model and path loss.

\section{A. Post-Disaster Communication Technique}

The D2D communication underlying the cellular network is executed by initiating the D2D peer discovery according to 3GPP Rel-13. During this process, large number of physical sidelink control signals are exchanged to initiate the discovery, synchronization, relaying etc. In post-disaster scenario, where there is significantly limited backup power supply to user devices, such D2D communication may not be feasible from the energy efficiency perspective. Therefore, users initiate the D2D discovery process only when cellular SNIR is sharply deteriorated. Therefore, our aim in this paper is to obtain the average success probability for either cellular or D2D communication in the post-disaster phase.

In normal communication scenario, a mobile device can connect to multiple networks, for instance, a mobile phone can have cellular, Wi-Fi and femtocell network simultaneously. However, this is not an effective communication method during the post-disaster phase due to the higher energy efficiency requirement and there is also very limited backup power supply available. In this paper, we propose and study the network performance when cellular and D2D communication are mutually exclusive for each user inside the disaster zone. Such a technique can be enforced by mobile network operators (MONs) when disaster hits the wireless network infrastructure. It eventually minimizes the control packet exchange which ultimately lowers the energy consumption and processing information in both user terminals and BSs.

Theorem 3. The network selection events will be independent as well as mutually exclusive when the user terminals are enforced to communicate in single mode, in either cellular or D2D communication, during the post-disaster scenario.

Proof. see Appendix B.

According to Theorem 3, the choice of communication mode not only lowers the energy consumption in the network but also increases the fairness on resource allocation among the users during the fragile stage of communication network. Therefore, considering the fact $\mathbb{P}\left(C_{t}: t \geq 0\right) \cdot \mathbb{P}\left(D_{t}: t \geq\right.$

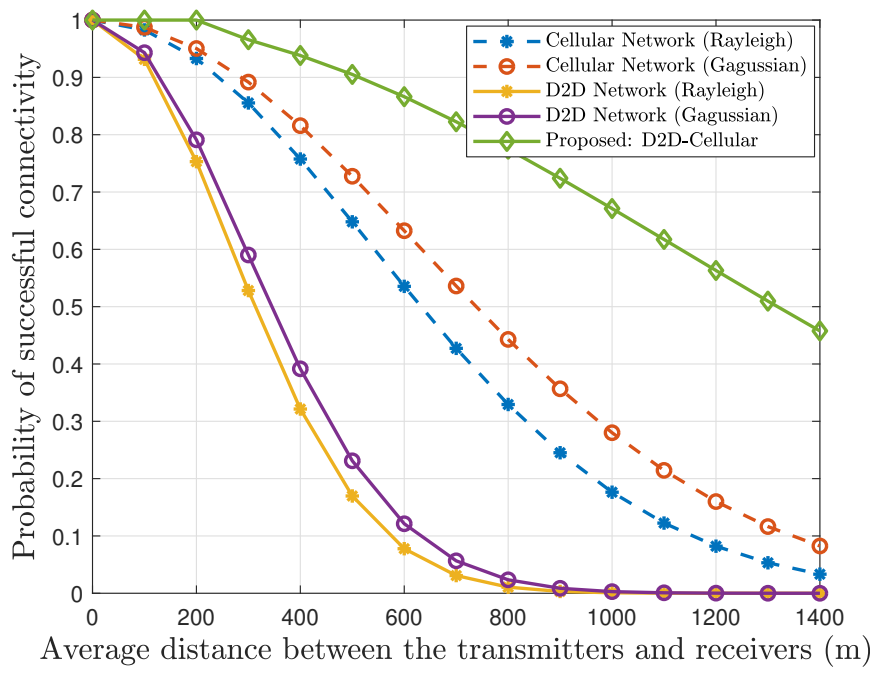

Fig. 2. The network coverage probability analysis for independent D2D, cellular network and the proposed network access method during the postdisaster scenarios.

$0)=0$, the communication success probability in the proposed method is obtained as following.

$$
\mathbb{P}(\mathbf{C} \cup \mathbf{D})=\mathbb{P}\left(C_{t}: t \geq 0\right)+\mathbb{P}\left(D_{t}: t \geq 0\right)
$$

Here, $\mathbb{P}(\mathbf{C} \cup \mathbf{D})$ is bounded to 1 when the BSs are phasing out. However, when the network comes towards the original state, $\mathbb{P}(\mathbf{C} \cup \mathbf{D})$ does not bound to 1 in which case Theorem 3 will no longer be enforced by MNOs.

\section{Performance Analysis}

In this section, the performance of the network is evaluated considering various post-disaster communication scenarios, for instance, BS thinning, network recovery process, and formation of D2D links. The Rayleigh fading channel model is considered with the thermal noise of $-105 \mathrm{dBm}$ and path loss exponent 3.68. The SNIR thresholds are taken to be $5 \mathrm{~dB}$ and $10 \mathrm{~dB}$ for user equipment and BS, respective, and the average transmit power, i.e., EIRP, of $23 \mathrm{dBm}$ and $46 \mathrm{dBm}$, respectively. The BSs and user terminals are distributed according to the PPP model with densities $\lambda_{b}$ and $\lambda_{u}$, respectively. All other network parameters are taken according to $3 \mathrm{GPP}$ and WINNER-II models.

Here, we study the behavior of post-disaster wireless network in cases the BSs are phasing out which is determined by thinning factor $p \in[0,1]$, where $p=0$ is the worst case scenario where no BSs are functioning and only D2D links are available for proximity service communication. First of all, we study the cellular communication without newly added BS or D2D communication pair. Secondly, the performance of the proposed network recovery methods will be investigated. The connectivity of the network is primarily measured due to the fact that the network connectivity is more important than network throughput during post-disaster phase from public safety perspective. 


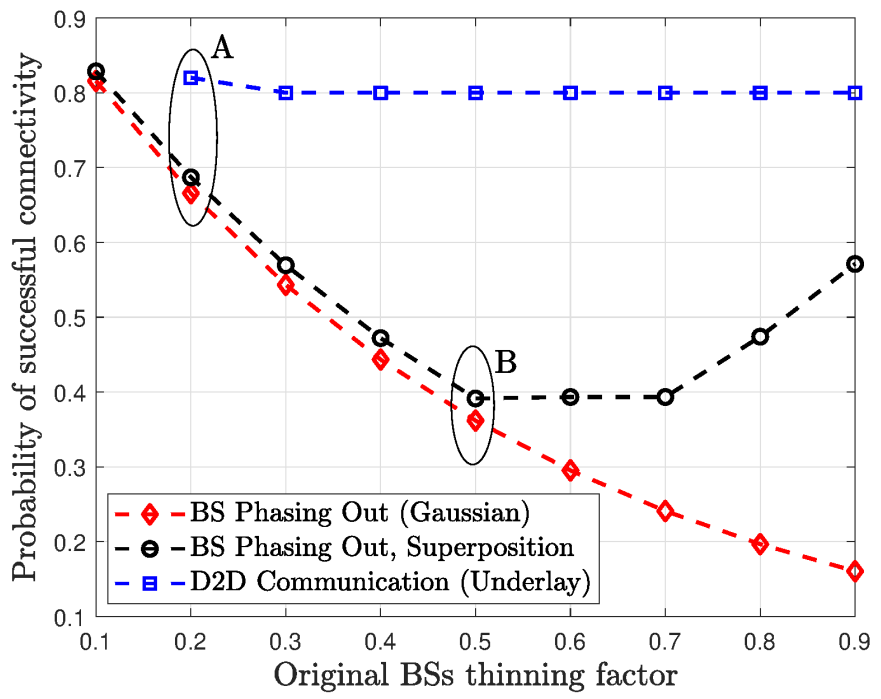

Fig. 3. The network coverage probability analysis for D2D communication in underlay mode, cellular communication with BS phasing out and BS superposition during the post-disaster scenarios.

As shown in Fig. 2, when there are no phasing out BSs, the coverage probability of cellular network decreases when the transmitter to receiver distance increases. Similarly, the coverage probability of D2D communication within the similar user distribution sharply decreases and reaches approximately null when the average distance is about $500 \mathrm{~m}$ due to the lower transmit power, higher D2D path-loss and fading effects. In this scenario, the D2D and cellular communications are independently working which indicates that there is presence of interference between cellular and D2D networks.

It is obvious that the coverage probability of Rayleigh fading channel is lower than Gaussian channels due to multiple fading states. When the disaster occurs, BSs thinning probability, $p$, is set to be 0.5 , indicating half of the BSs are dysfunctional or partly functioning. At the same time, $40 \%$ of total users initiate the D2D communication independently. When the proposed algorithm is implemented, it can be observed in the Fig. 2 that the overall coverage probability will be significantly improved such that a large number of users get temporary coverage during post-disaster. The complexity of the proposed algorithm is very low because users have to measure the received SNIR only from cellular downlink signal to decide whether it should initiate the device detection for possible D2D communication or not.

The performance in terms of network connectivity in the proposed post-disaster scenario has been presented in Fig. 3 . When the BSs are phasing out, it is obvious that the probability of a user within the network coverage range decreases under any channel fading condition as shown in the figure. For instance, when $20 \%$ BSs are phasing out, approximately $70 \%$ cellular users can communicate with a predefined SNIR, whereas when BSs phasing out rate is doubled, the coverage probability further decreases to $45 \%$. The coverage degradation rate is almost similar for both fading and non-facing wireless channels.

The new BSs are distributed, according to PPP with parameter $\lambda>0$, to provide the communication services to the disconnected users as indicated by point B in Fig. 3. At this instant of post-disaster network, the channel fading is considered to be the Rician distribution assuming the flying BS, or unmanned aerial vehicles (UAVs), provide such coverage where there is a better chance of line-of-sight communications [15]. As shown in the Fig. 3, when BSs superposition occurs at point $\mathrm{B}$, the user coverage probability can be improved from 0.4 to 0.6 . However, it primarily depends on the number of added BSs and their distribution patterns. Similarly, the D2D communication is enabled when BSs phasing out occurs at $20 \%$, D2D communication, being independent of the cellular communication, remains constant at around $80 \%$ irrespective of BSs thinning process as described in the previous section.

\section{CONCLUSION}

Since wireless communication infrastructure plays very important role in public safety during post-disaster scenario to save more lives, the analytical studies of such cases are critically imperative for $5 \mathrm{G}$ and beyond. In this paper, we analyzed the network behavior during a typical post-disaster scenario where BSs undergo the thinning process due to the damage to the network infrastructure. During network recovery phase, we also investigated the network performance when new temporary aerial or ground-based BSs are deployed which transform the network into the BS superposition phase. Furthermore, D2D assisted cellular communication helps to increase the link level network connectivity which is highly appreciated during post-disaster phase because the network coverage is more desirable than the network throughout in such cases. We also described the analytical study of D2D and cellular communication in underlay mode and revealed that it can effectively increase the network coverage.

\section{Appendix A: Proof of Theorem 2}

Proof. Here, we consider the interference model for both BSs phasing out and superposition processes. Let $I_{1}=$ $\sum_{x \in \Phi_{A}} P_{L}(x, y)$ is the aggregate interference power at the random user, where $\Phi_{A}$ is the set of locations of active BSs and $P_{L}(x, y)$ is the average path loss function between points $x$ and $y$. Similarly, When network superposition occurs, either due to added BSs or new D2D link formation, the interference to the user at point $x$ is $I_{2}=\sum_{k=1}^{K} \sum_{x \in \Phi_{k}} P_{L, k}(x, y)$. The interference to a particular user irrespective of network mode of operation, the total interference is

$$
I=I_{1}+I_{1}=\sum_{x \in \Phi_{A}} P_{L}(x, y)+\sum_{k=1}^{K} \sum_{x \in \Phi_{k}} P_{L, k}(x, y) .
$$

The Laplace transform of (10) is obtained as following.

$$
I(s)=E_{\Phi_{A}}\left[\mathrm{e}^{-s \sum_{x \in \Phi_{A}} P_{L}(x)}\right] \cdot \prod_{k=1}^{K} E_{\Phi_{k}}\left[\mathrm{e}^{-s \sum_{x \in \Phi_{k}} P_{L, k}(x)}\right],
$$


which can be reduced to

$$
\begin{aligned}
I(s)=\exp (-p \lambda & \int_{\mathbb{R}^{2}}\left[1-\mathrm{e}^{-s P_{L}(x)}\right] d x \\
& \left.\quad-\sum_{k=1}^{K} \lambda_{k} \int_{\mathbb{R}^{2}}\left[1-\mathrm{e}^{-s P_{L, k}(x)}\right] d x\right) .
\end{aligned}
$$

Here, $P_{L}(x)$ and $P_{L, k}(x)$ follow PPP. There are two new networks in post-disaster phase, i.e., $k=1,2$, for new D2D networks and added cellular networks. Therefore, based on the superposition property of PPP, we can generalize $P_{L}(x)$, $P_{L, 1}(x)$ and $P_{L, 2}(x)$ into a single link vector. Then, (12) becomes

$$
I(s)=\exp \left(-\left[p \lambda+\sum_{k=1}^{K} \lambda_{k}\right] \int_{\mathbb{R}^{2}}\left[1-\mathrm{e}^{-s \mathbf{P}_{\mathbf{L}}(\mathbf{x})}\right] d x\right)
$$

which is a PPP distribution with new parameter $\left(p \lambda+\sum_{k=1}^{K} \lambda_{k}\right)$. This completes the proof.

\section{ApPEndix B: PRoOf OF THEOREM 3}

Proof. Assuming $\mathbf{C}=\left(C_{t}: t \geq 0\right)$ is the PPP which undergoes thinning when disaster occurs and $\mathbf{D}=\left(D_{t}: t \geq 0\right)$ is the formation of D2D pairs which is superimposed within the same cellular network area during post-disaster phase. As shown in Theorem 1, counting processes $\mathbf{C}$ and $\mathbf{D}$ are independent to each other. According to the proposed method, the selection of communication mode is mutually exclusive. Now, we will find the condition in which this is true. Since they are independent events, the following is true.

$$
\mathbb{P}\left(C_{t}: t \geq 0 \cap D_{t}: t \geq 0\right)=\mathbb{P}\left(C_{t}: t \geq 0\right) \cdot \mathbb{P}\left(D_{t}: t \geq 0\right)
$$

Since selection mode is assumed to be mutually exclusive,

$$
\mathbb{P}\left(\left(C_{t}: t \geq 0\right) \cap \mathbb{P}\left(D_{t}: t \geq 0\right)\right)=0 .
$$

According to (14) and (15), following should be true.

$\mathbb{P}\left(C_{t}: t \geq 0 \cap D_{t}: t \geq 0\right)=\mathbb{P}\left(C_{t}: t \geq 0\right) \cdot \mathbb{P}\left(D_{t}: t \geq 0\right)=0$

Therefore, $\mathbb{P}\left(C_{t}: t \geq 0\right) \cdot \mathbb{P}\left(D_{t}: t \geq 0\right)=0$ is true only when i) $\mathbb{P}\left(C_{t}: t \geq 0\right)=0$ if $\mathbb{P}\left(D_{t}: t \geq 0\right) \neq 0$, ii) $\mathbb{P}\left(D_{t}:\right.$ $t \geq 0)=0$ if $\mathbb{P}\left(C_{t}: t \geq 0\right) \neq 0$, or iii) $\mathbb{P}\left(C_{t}: t \geq 0\right)=$ $\mathbb{P}\left(D_{t}: t \geq 0\right)=0$. During the post-disaster phase, When user terminals are enforced to choose one mode of communication, then two events will be independent and mutually exclusive.

This completes the proof.

\section{ACKNOWLEDGMENT}

This work was supported by the UK Engineering \& Physical Sciences Research Council (EPSRC): Project DARE under grant agreement no. EP/P028764/1.

\section{REFERENCES}

[1] T. Sakano, S. Kotabe, T. Komukai, T. Kumagai, Y. Shimizu, A. Takahara, T. Ngo, Z. M. Fadlullah, H. Nishiyama, and N. Kato, "Bringing movable and deployable networks to disaster areas: development and field test of MDRU," IEEE Network, vol. 30, no. 1, pp. 86-91, 2016.

[2] M. Li, H. Nishiyama, N. Kato, Y. Owada, and K. Hamaguchi, "On the energy-efficient of throughput-based scheme using renewable energy for wireless mesh networks in disaster area," IEEE Trans. Emerg. Topics Comput., vol. 3, no. 3, pp. 420-431, Sept 2015.

[3] "3rd Generation Partnership Project: Technical specification group services and system aspects; enhancements for multimedia priority service (MPS) (Release 11)," https://portal.3gpp.org/desktopmodules/ Specifications/SpecificationDetails.aspx?specificationId=934, 2011, accessed: 2017-11-03

[4] J. Zhao, Y. Liu, K. K. Chai, Y. Chen, and M. Elkashlan, "Joint subchannel and power allocation for noma enhanced d2d communications," IEEE Trans. Commun., vol. 65, no. 11, pp. 5081-5094, Nov 2017.

[5] A. Alnoman and A. Anpalagan, "On D2D communications for public safety applications," in 2017 IEEE Canada International Humanitarian Technology Conference (IHTC), July 2017, pp. 124-127.

[6] K. Ali, H. X. Nguyen, Q. T. Vien, P. Shah, and Z. Chu, "Disaster management using D2D communication with power transfer and clustering techniques," IEEE Access, vol. 6, pp. 14643-14 654, 2018.

[7] A. Al-Hourani, S. Kandeepan, and A. Jamalipour, "Stochastic geometry study on Device-to-Device communication as a disaster relief solution," IEEE Trans. Veh. Technol., vol. 65, no. 5, pp. 3005-3017, May 2016.

[8] H. Nishiyama, M. Ito, and N. Kato, "Relay-by-smartphone: realizing multihop device-to-device communications," IEEE Commun. Mag., vol. 52, no. 4, pp. 56-65, April 2014.

[9] A. He, L. Wang, Y. Chen, K. K. Wong, and M. Elkashlan, "Spectral and energy efficiency of uplink D2D underlaid massive MIMO cellular networks," IEEE Trans. Commun., vol. 65, no. 9, pp. 3780-3793, Sept 2017.

[10] S. Kusaladharma and C. Tellambura, "Performance characterization of spatially random energy harvesting underlay D2D networks with transmit power control," IEEE Trans. on Green Commun. and Net., vol. 2, no. 1, pp. 87-99, March 2018.

[11] M. Banagar, B. Maham, P. Popovski, and F. Pantisano, "Power distribution of device-to-device communications in underlaid cellular networks," IEEE Wireless Commun. Lett., vol. 5, no. 2, pp. 204-207, April 2016.

[12] D. G.C. and K. Navaie, "A low-latency zone-based cooperative spectrum sensing," IEEE Sensors Journal, vol. 16, no. 15, pp. 6028-6042, Aug 2016.

[13] A. M. Hayajneh, S. A. R. Zaidi, D. C. McLernon, and M. Ghogho, "Drone empowered small cellular disaster recovery networks for resilient smart cities," in 2016 IEEE Intl. Conf. on Sensing, Communication and Networking (SECON Workshops), June 2016, pp. 1-6.

[14] M. Z. Win, P. C. Pinto, and L. A. Shepp, "A mathematical theory of network interference and its applications," Proceedings of the IEEE, vol. 97, no. 2, pp. 205-230, Feb 2009.

[15] M. M. Azari, F. Rosas, K. Chen, and S. Pollin, "Optimal UAV positioning for terrestrial-aerial communication in presence of fading," in 2016 IEEE Global Comm. Conf. (GLOBECOM), Dec 2016, pp. 1-7. 